\title{
Characterization of Brain Plasticity in Schizophrenia Using Template Deformation
}

\author{
Abraham Dubb, Zhiyong Xie, Ruben Gur, Raquel Gur, and James Gee \\ Departments of Bioengineering, Psychiatry and Radiology \\ University of Pennsylvania \\ Philadelphia, PA, USA 19104 \\ $\{$ adubb, zxie\}@grasp. cis. upenn.edu \\ $\{$ gur, raquel\}@bbl.med.upenn.edu \\ gee@rad.upenn.edu
}

\begin{abstract}
Abnormal neurodevelopment may play a role in the pathophysiology of schizophrenia. We used Template Deformation Morphometry (TDM) to examine point-wise age-related changes in patients with schizophrenia compared with healthy brains. We used a set of skullstripped brains from an image database of cranial MRIs. We then deformed a template image to the rest of the brains creating a set of deformations. Using the Jacobians of these deformations, we calculated the voxel-wise $t$-scores for comparison of controls with patients. We also calculated the voxel-wise Pearson correlation of Jacobian with age for both controls and patients. By examining the volume renderings of these statistical fields, we found that healthy people undergo age-related expansion of the ventricles and surrounding periventricular white matter. In contrast, patients show much less of this age-related expansion. We conclude that TDM demonstrates altered developmental course in the brains of patients with schizophrenia.
\end{abstract}

\section{Introduction}

The study of schizophrenia has benefited from the advent of high resolution MRI and advanced morphometry techniques. Investigators have reported volumetric differences in multiple regions including the frontal and temporal lobes, ventricles, hippocampus and extra-pyramidal structures 12345. The existence of morphologic differences has motivated evaluation of the developmental course of neuroanatomic measures to assess whether neurodevelopment or neurodegeneration may play a role in schizophrenia. Several studies reported regional age-related changes of the brain 67,8 . While these studies have produced conflicting results, there is a growing consensus that brain plasticity is altered in schizophrenia, underscoring the importance of studying age-related changes in neuroanatomy 6 .

To examine brain morphometry, we applied Template Deformation Morphometry (TDM), a relatively novel method. TDM, described in detail in previous studies 910, provides a voxel-wise statistical description of a set of volumetric images. Normally, voxel-based morphometry techniques have reported 
point-wise $t$-scores comparing two clinical populations. In order to compare agerelated changes in brain volume between controls and schizophrenia patients, we calculated the point-wise Pearson correlation coefficient relating age to each Jacobian value for both groups. In this way, we are able to reduce an entire set of volumetric images down to a single statistical parametric map describing the age-related size change, expansion or contraction, at each voxel.

\section{Methods}

\subsection{Subjects and Data Acquisition}

The Schizophrenia Center at the University of Pennsylvania maintains a database containing cranial MRIs of both psychiatric patients and healthy volunteers. We selected right handed participants with a diagnosis of schizophrenia and healthy controls for whom a $1 \mathrm{~mm}$ slice thickness scan was available. Magnetic resonance imaging scans were acquired on a $1.5 \mathrm{~T}$ scanner (Signa; General Electric Co., Milwaukee, WI) with a spoiled gradient recalled pulse sequence using the following parameters: flip angle of $35^{\circ}$, repetition time of $35 \mathrm{~ms}$, echo time of $6 \mathrm{~ms}$, field of view of $24 \mathrm{~cm}, 1$ repetition, $1 \mathrm{~mm}$ slice thickness and no interslice gaps. Transaxial images were in planes parallel to the orbitomeatal line, with resolution of $0.9375 \times 0.9375 \mathrm{~mm}^{2}$.

\subsection{Brain Extraction}

The skull, scalp, and other extra-cranial tissues were removed from each image using the software, Brain Surface Extraction Program, developed by Shattuck, et al [11 12. In most cases, the software performed perfectly and extracted the brain without error. In several cases, however, additional manual editing was required to remove retained skull tissue.

\subsection{Image Registration}

The registration method we applied is a spline-based extension to Thirion's Demons registration [13. It uses optical flow to determine the correspondence of voxels which exhibit sufficiently large intensity gradients. Based on the estimated correspondences, a B-spline function over the whole brain volume is determined using weighted scattered data approximation. This two-step algorithm is applied over multiple resolution levels in conventional coarse to fine fashion: both the resolution of the images and the number of spline control parameters are simultaneously adjusted. Specifically, starting with B-spline functions that have a small number of parameters, the algorithm is iterated to match the coarse features of the images. The result is used to initialize the registration at the next resolution level, where the number of spline parameters is increased to allow alignment of the finer features that are apparent in the higher resolution images. This strategy provides a way to incrementally refine the registration and improves the robustness of the method. We repeated this registration for each subject, creating a deformation field $F$ that mapped the template to each subject image. 


\subsection{Statistical Analysis of the Jacobian}

The deformation field is defined as a set of $N$ displacement vectors where $N$ is the number of voxels composing the template brain. The following equation

$$
\mathbf{u}_{k}=\left(u_{k}^{1}, u_{k}^{2}, u_{k}^{3}\right)
$$

gives the displacement vector, composed of the orthogonal displacements, $u_{k}^{1}, u_{k}^{2}$, $u_{k}^{3}$, needed to map the $k^{\text {th }}$ voxel of the template to the corresponding voxel of the patient. Using the vector field, $\mathbf{u}_{i}$, which describes the displacement field that relates the template to the subject brain, $i$, we can generate the following transformation equation:

$$
T_{i}(\mathbf{x})=\mathbf{x}+\mathbf{u}_{i}(\mathbf{x}) .
$$

$T_{s}(\mathbf{x})$ gives the corresponding position in subject brain $i$ for voxel $\mathbf{x}$ in the template. In order to describe the expansion or contraction that occurs at each voxel in this transformation, we use the following quantity:

$$
\left|\frac{\partial T_{i}}{\partial \mathbf{x}}\right|
$$

which is the determinant of the Jacobian of the transformation. For ease of notation, we will simply refer to this value as the Jacobian or $J . J_{k}$ values provide a regional measure of size of the subject in relation to the template by providing a measure of vector "splay" of the deformation field at each voxel. In order to normalize the Jacobian for global variation [9], we scale this quantity at each voxel, $k$, by the sum of all Jacobian values in the image:

$$
j_{k}=\frac{J_{k}}{\sum_{i=0}^{N} J_{i}} .
$$

Now that our set of volumetric images have been converted into corresponding normalized Jacobian fields, we may perform our voxel-wise statistical calculations. As is traditional in voxel based morphometry studies, we use $\log j_{k}$ to generate our statistical parametric maps (SPM). For ease of notation, we will refer to $\log j_{k}$ as simply $j_{k}$. In this study, we generated two types of SPMs: $t$-scores and Pearson scores. The first type of SPM compares the average magnitude of $j_{k}$ at each voxel using the following equation:

$$
t_{k}=\frac{\overline{j_{k}^{c}}-\overline{j_{k}^{s}}}{\sigma_{k} \sqrt{\frac{1}{n_{s}}+\frac{1}{n_{c}}}},
$$

where

$$
\sigma_{k}=\sqrt{\frac{\left(n_{c}-1\right) \sigma_{c}^{2}+\left(n_{s}-1\right) \sigma_{s}^{2}}{n_{c}+n_{s}-2}} .
$$

$\overline{j_{k}^{c}}$ and $\overline{j_{k}^{s}}$ are the mean normalized Jacobians for the control and schizophrenia groups at the $k^{t h}$ voxel, respectively. $\sigma_{c}$ and $\sigma_{s}$ are the standard deviations of 
Table 1. Demographic characteristics of the study populations.

\begin{tabular}{|l|c|c|}
\hline & Control & Schizophrenia \\
\hline$n$ & 78 & 36 \\
female & 43 & 12 \\
age range & $18-84$ & $18-64$ \\
mean age (S.D.) & $30.5(13.0)$ & $30.7(10.8)$ \\
\hline
\end{tabular}

$j_{k}$ for controls and patients, respectively. $n_{c}$ and $n_{s}$ are the number of subjects in the control group and schizophrenia group. A positive value for $t_{k}$ signifies a larger average $j_{k}$ value (and thus, larger regional size) in the control population while a negative $t_{k}$ suggests the opposite. The $t$ field may be viewed as a regional size comparison between the two groups

In order to test the hypothesis of altered age-related changes in brain morphology in schizophrenia, we need to incorporate age into our statistical measure. To do this we calculate the point-wise Pearson correlation, $r_{k}$, between $j_{k}$ and age:

$$
r_{k}=\frac{S P}{\sqrt{S S_{a} S S_{j_{k}}}}
$$

where

$$
\begin{gathered}
S P=\sum_{i=0}^{n} a(i) j(i)_{k}-\frac{\sum_{i=0}^{n} a(i) \sum_{i=0}^{n} j(i)_{k}}{n}, \\
S S_{a}=\sum_{i=0}^{n} a(i)^{2}-\frac{\left(\sum_{i=0}^{n} a(i)\right)^{2}}{n},
\end{gathered}
$$

and

$$
S S_{j_{k}}=\sum_{i=0}^{n} j(i)_{k}^{2}-\frac{\left(\sum_{i=0}^{n} j(i)_{k}\right)^{2}}{n} .
$$

$n$ is the number of subjects in the population, $j(i)_{k}$ is the log of the normalized Jacobian at the $k$ th voxel in the $i$ th subject. $a(i)$ is the age, in years, of subject $i$. Positive values for $r_{k}$ reflect a positive relationship between age and $j_{k}$ and hence, regional age-related expansion, while negative $r_{k}$ signifies regional decrease with age. Pearson fields were generated for both the control population and the patient population.

Both $t$ and Pearson fields are presented as color volumes, in which blue represents negative values and red represents positive values. Because a threedimensional color volume cannot be displayed in its entirety due to space limitations, we chose to include several representative projections that best portray the differences between the two populations. 

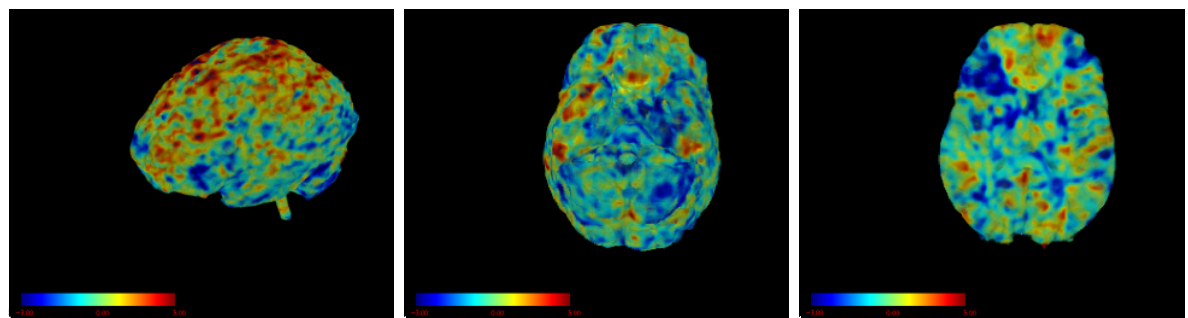

Fig. 1. Voxel-wise $t$-score map comparing healthy controls with schizophrenia patients. Red areas reflect regions of expansion in the control population, and areas of blue reflect regions of expansion in the patient population.
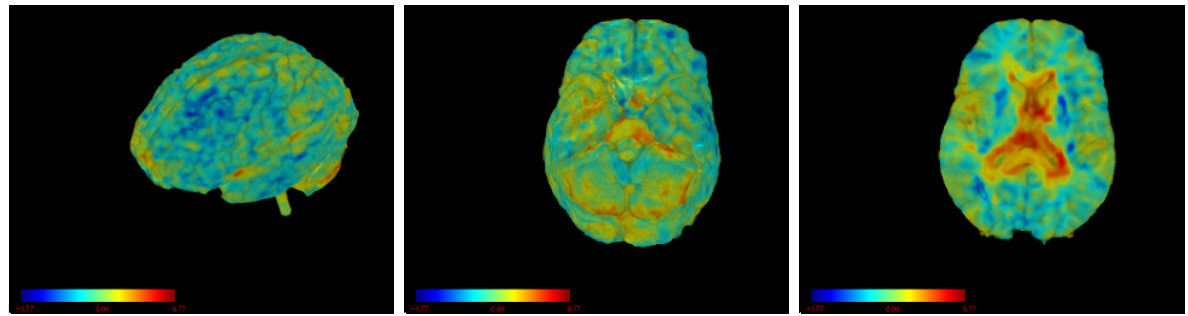

Fig. 2. Voxel-wise Pearson score depicting age-related changes of the Jacobian in control subjects. Red means expansion with age while blue means contraction.
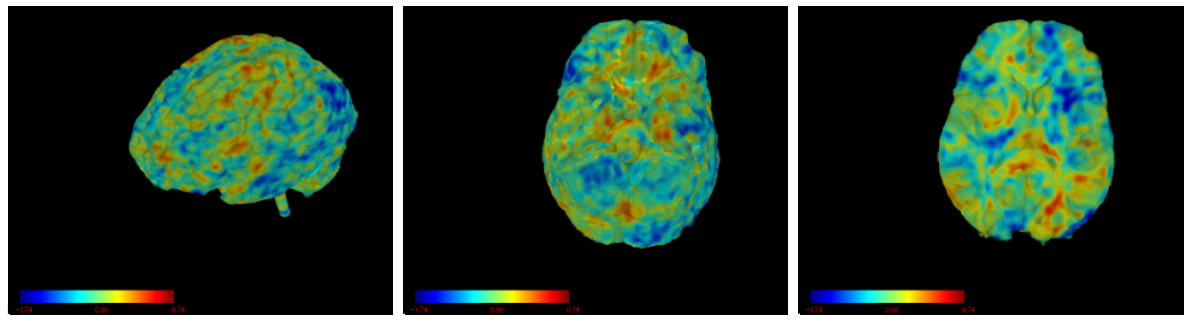

Fig. 3. Voxel-wise Pearson score depicting age-related changes of the Jacobian in schizophrenia patients. Red means expansion with age while blue means contraction.

\section{Results}

Our query of the Schizophrenia Center database yielded a total of 114 cranial MRIs, 78 of control and 36 of patients with schizophrenia. Table 1 shows the basic demographic characteristics of the two populations. Figure 1 shows the $t$-score map for the comparison between controls and patients. Note how the frontal and parietal areas are larger in controls while temporal and ventral structures are larger in the patient population. Age-related changes in the control population are shown in figure 2. Notice the intense area of expansion in the ventricles and periventricular white matter. This pattern of neurodevelopment 
is in stark contrast to the age-related pattern in the patient population, depicted in figure 3, which shows much less age-related expansion in the ventricles and peri-ventricular white matter. These differences persisted even after we repeated the study with a different template.

\section{Discussion}

Using template deformation morphometry and an age-dependent statistical metric, we are able to show reduced age-related expansion of the ventricles and periventricular white matter of patients with schizophrenia. We previously showed substantially reduced age-related changes in the corpus callosum of patients versus controls [10]. Other investigators have suggested that periventricular leukomalacia may predate schizophrenia and play a causative role [14 15]. While speculative, the hypothesis is supported by a recent study reporting failure of normal age-related white matter expansion in schizophrenia [16]. The extent to which abnormalities in time-dependent neuroanatomic changes are prominent in schizophrenia merits further study [17.

Our study has several limitations. First, we assume that brains from multiple subjects may be used to create a voxel-wise model of age dependency. The process of aging may be highly individual creating uncertainty in the voxel-wise statistics. Second, there are certain limitations with our registration algorithm. As a fully-automated procedure, the deformation cannot be expected to generate a perfect anatomical correlation between template and subject. Thus, what we gain in speed we lose in accuracy. Nonetheless, the method has been validated for relatively large substructures [18] and we have tried to restrict the findings we report to only those gross features which the method could be expected to detect.

We believe that TDM is a powerful and scalable procedure for detecting both static structural differences and time-dependent changes in clinical populations. In future work, we plan to incorporate expert-identified correspondences into our registration algorithm, increasing the accuracy of our deformation, as well as the detail of the results we present.

Acknowledgements. This work was supported in part by the USPHS under grants NS-33662, LM-03504, MH-62100, AG-15116, AG-17586 and DA-14418.

\section{References}

1. RE. Gur, PE. Cowell, A. Latshaw, BI. Turetsky, RI. Grossman, SE. Arnold, WB. Bilker, and RC. Gur. Reduced dorsal and orbital prefrontal gray matter volumes in Schizophrenia. Archives of General Psychiatry, 57(8):761-8, 2000.

2. RE. Gur, BI. Turetsky, PE. Cowell, C. Finkelman, V. Maany, RI. Grossman, SE. Arnold, WB. Bilker, and RC. Gur. Temporolimbic volume reductions in Schizophrenia. Archives of General Psychiatry, 57(8):769-75, 2000. 
3. W. Cahn, HE. Pol, M. Bongers, HG. Schnack, RC. Mandl, NE. Van Haren, S. Durston, H. Koning, JA. Van Der Linden, and RS. Kahn. Brain morphology in antipsychotic-naive Schizophrenia: A study of multiple brain structures. British Journal of Psychiatry - Supplementum, 43:s66-72, 2002.

4. RE. Gur, V. Maany, D. Mozley, C. Swanson, W. Bilker, and RC. Gur. Subcortical MRI volumes in neuroleptic-naive and treated patients with Schizophrenia. American Journal of Psychiatry, 155:1711-1717, 1998.

5. MS. Keshavan, D. Rosenberg, JA. Sweeney, and JW. Pettegrew. Decreased caudate volume in neuroleptic-naive psychotic patients. American Journal of Psychiatry, 155(6):774-8, 1998.

6. LE. Delisi. Regional brain volume change over the life-time course of Schizophrenia. Journal of Psychiatric Research, 33:535-541, 1999.

7. BT. Woods, D. Yurgelun-Todd, FM. Benes, FR. Frankenburg, Jr. Pope HG, and J. McSparren. Progressive ventricular enlargement in Schizophrenia: comparison to bipolar affective disorder and correlation with clinical course. Biological Psychiatry, 27(3):341-52, 1990.

8. JL. Rapoport, J. Giedd, S. Kumra, L. Jacobsen, A. Smith, P. Lee, J. Nelson, and S. Hamburger. Childhood-onset Schizophrenia. progressive ventricular change during adolescence. Archives of General Psychiatry, 54(10):897-903, 1997.

9. D. J. Pettey and J. C. Gee. Sexual dimorphism in the corpus callosum: A characterization of local size variations and a classification driven approach to morphometry. NeuroImage, 17:1504-1511, 2002.

10. A. Dubb, B. Avants, R. Gur, and J. C. Gee. Shape characterization of the corpus callosum in Schizophrenia using template deformation. In Takeyoshi Dohi and Ron Kikinis, editors, MICCAI, pages 381-388. Springer, Berlin, 2002.

11. DW. Shattuck, SR. Sandor-Leahy, KA. Schaper, DA. Rottenberg, and RM. Leahy. Magnetic resonance image tissue classification using a partial volume model. Neuroimage, 13(5):856-76, 2001.

12. S. Sandor and R. Leahy. Surface-based labeling of cortical anatomy using a deformable atlas. IEEE Transactions on Medical Imaging, 16(1):41-54, 1997.

13. JP. Thirion. Image matching as a diffusion process: an analogy with Maxwell's demons. Medical Image Analysis, 2(3):243-60, 1998.

14. JH. Gilmore, M. Castillo, and M. Rojas. Early onset Schizophrenia in a patient with premature birth, germinal matrix hemorrhage and periventricular leukomalacia. Schizophrenia Research, 44(2):158-60, 2000.

15. KL. Thomas and M. Huttunen. Neural migration, pro-inflammatory interleukins and periventricular leukomalacia: involvement in Schizophrenia. Trends in Neurosciences, 22(9):389-90, 1999.

16. G. Bartzokis. Schizophrenia: Breakdown in the well-regulated lifelong process of brain development and maturation. Neuropsychopharmacology, 27(4):672-83, 2002.

17. LE. DeLisi. Is Schizophrenia a lifetime disorder of brain plasticity, growth and aging? Schizophrenia Research, 23:119-129, 1997.

18. Z. Xie, Lydia Ng, and J. C. Gee. Two algorithms for non-rigid image registration and their evaluation. In SPIE, 2003, in press. 